\title{
AVALIAÇÃO E CARACTERIZAÇÃO DA PASTILHA SENSORA DE UM ELETRODO DE SEGUNDA ESPÉCIE SENSÍVEL A OXALATO
}

\author{
A. C. VILLAFRANCA ${ }^{1}$; M. de MORAES ${ }^{1 *}$; L. PEZZA ${ }^{2}$
}

Resumo:

O eletrodo $\mathrm{Pt}|\mathrm{Hg}| \mathrm{Hg}_{2} \mathrm{Ox} \mid \mathrm{Grafite}\left(\mathrm{Ox}=\right.$ íon oxalato) foi estudado utilizando $\mathrm{NaClO}_{4}$ como eletrólito de suporte. Foram feitas experiências preliminares utilizando o $\mathrm{NaNO}_{3}$ como eletrólito de suporte seguindo a mesma metodologia do trabalho anterior e verificou-se que havia uma diminuição na sensibilidade do eletrodo em função do tempo de uso. Visando compreender a diferença de comportamento do eletrodo em meio de $\mathrm{NaClO}_{4}$ e $\mathrm{NaNO}_{3}$ foram feitas experiências de obtenção de curvas de potencial em função do tempo para verificar seu comportamento em função do tempo e em diferentes meios iônicos. A morfologia da superfície da pastilha sensora usada em meio de $\mathrm{NaNO}_{3}$ apresentou alteração, a qual sugere que este meio favorece reações na superfície da pastilha sensora que fica em contato com a solução.

Palavras-chave: eletrodo de segunda espécie, oxalato, perclorato, nitrato

\section{Introdução}

Compostos contendo o íon oxalato são objeto de muitos estudos nos mais variados campos da ciência. Contínuas investigações vêm sendo feitas enfocando este íon, bem como seus sais insolúveis. A grande demanda está principalmente no campo da medicina, laboratórios de análises clínicas e nas indústrias farmacêutica e de alimentos, tornando muito importante os estudos relacionados com o comportamento de sistemas contendo o íon oxalato e seus sais [6].

A maioria dos procedimentos de análise de oxalato são, muitas vezes, complicados e/ou dispendiosos devido aos reagentes e aparelhos necessários $[2,8,9,10,13]$. A detecção do oxalato é feita de forma indireta em muitos casos.

A potenciometria, como técnica analítica, tem uma série de vantagens, porque sua instrumentação é relativamente simples, ainda que em diferentes condições específicas de determinações analíticas. De um lado esta técnica permite determinar substâncias em solução em um grande intervalo de concentração usando um mesmo equipamento, apenas modificando ligeiramente o procedimento analítico. Por outro lado, medidas potenciométricas podem ser executadas sobre condições de laboratório e de campo. Estas são convenientes para controle contínuo ou remoto da concentração analítica em plantas industriais e para monitoramento ambiental [12].

\footnotetext{
${ }^{1}$ Departamento de Química Analítica - Instituto de Química - Universidade Estadual Paulista - CEP 14800-900 - Araraquara - SP - Brasil ${ }^{2}$ Departamento de Química Orgânica - Instituto de Química - Universidade Estadual Paulista - CEP 14800-900 - Araraquara - SP - Brasil
} 
$\mathrm{Na}$ literatura são descritos alguns trabalhos envolvendo a construção e a utilização de eletrodos de membrana líquida [7] e de segunda espécie $[5,11]$ para a determinação potenciométrica de oxalato.

Em um estudo físico-químico anterior, nosso grupo de pesquisa desenvolveu um eletrodo $\mathrm{Pt}|\mathrm{Hg}| \mathrm{Hg}_{2} \mathrm{Ox} \mid \mathrm{Grafite}$, onde $\mathrm{Ox}=$ ín oxalato (Figura 1), utilizando $\mathrm{NaClO} 4$ como eletrólito de suporte [4]. Pensando numa possível aplicação analítica deste eletrodo, buscou-se a utilização de um meio iônico mais conveniente. Assim, optou-se pelo uso do NaNO3, pois a amostra poderia ser digerida em meio de $\mathrm{HNO} 3$ em vez de $\mathrm{HClO} 4$ (este, além de mais perigoso, é de custo mais elevado que o primeiro), sendo que após a neutralização do $\mathrm{HNO}_{3}$ esta estaria pronta para ser analisada em meio de $\mathrm{NaNO}_{3}$. Foram feitas experiências preliminares utilizando o $\mathrm{NaNO}_{3}$ como eletrólito de suporte seguindo a mesma metodologia do trabalho anterior, e verificou-se que havia uma diminuição na sensibilidade do eletrodo em função do tempo de uso em meio de $\mathrm{NaNO}_{3}$.

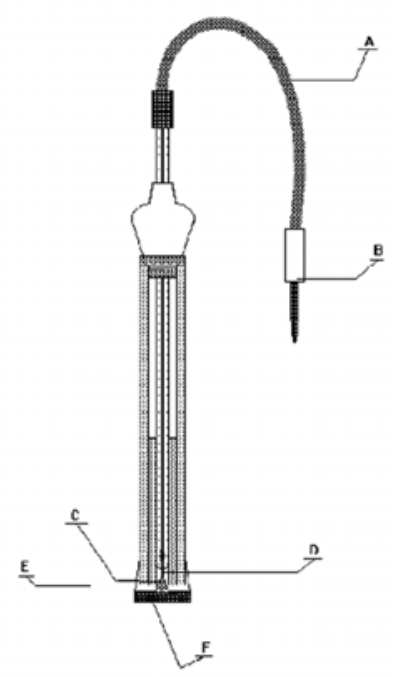

Figura 1. Eletrodo de oxalato mercuroso. A, cabo condutor; B, plug banana; C, mercúrio metálico; D, fio de $\mathrm{Pt}$; E, cola de silicone; F, pastilha sensora (Grafite $|\mathrm{Hg}| \mathrm{Hg}_{2} \mathrm{Ox}$ )

\section{Parte Experimental}

\section{Materiais}

Todos os reagentes utilizados na execução do presente trabalho foram de pureza analítica (p.a.). Foi utilizada água desionizada para o preparo das soluções de interesse. O mercúrio usado foi purificado e destilado em nosso laboratório [1]. Todas as operações foram realizadas em sala climatizada a $(25 \pm 1)^{\circ} \mathrm{C}$, onde encontram-se instalados os equipamentos de medida.

\section{Métodos}

\section{Curvas Potencial em função do Tempo}

Foram feitas experiências para verificar o comportamento do eletrodo na célula potenciométrica $\quad \mathrm{Ag}|\mathrm{AgCl}| \mathrm{Cl}^{-} \quad v s$. $\mathrm{Pt}|\mathrm{Hg}| \mathrm{Hg}_{2} \mathrm{Ox} \mid$ Grafite, em função do tempo e em diferentes meios iônicos. Utilizou-se o potenciostato Microquímica modelo MQPG-01 interfaceado a um microcomputador da linha PC compatível empregando software MQPG que controla as condições experimentais e armazena os dados experimentais.

\begin{tabular}{|c|c|c|c|c|}
\hline & $\begin{array}{c}\mid \mathrm{NaCl}_{(\mathrm{aw})}= \\
=0,010 \mathrm{~mol} \mathrm{~L}^{\prime}\end{array}$ & & $\begin{array}{c}{\left[\mathrm{N}_{2}(\mathrm{Ox}]_{\mathrm{Asc}_{\mathrm{C}}=\mathrm{x}}\right.} \\
\mathrm{mol} \mathrm{I.}\end{array}$ & \\
\hline$(-) \wedge \mathrm{g} \mid \wedge \mathrm{g} C \mathrm{Cl}$ & & $\begin{array}{l}{\left[Y^{\prime}\right]_{\text {tiw }}=} \\
=0.700 \\
\text { mol L' }\end{array}$ & & Gufitel $\mathrm{Hg}_{2} \mathrm{Ox} / \mathrm{Hg} / \mathrm{h}(+)$ \\
\hline & $\begin{array}{c}{[\mathrm{Y}]_{(x)}=} \\
=0.690 \mathrm{inol} \mathrm{t}^{-1}\end{array}$ & & $\begin{array}{c}{[Y]_{\text {Git }}=} \\
=(0,700-3 x) \mathrm{mol} \\
\mathrm{L}^{1}\end{array}$ & \\
\hline
\end{tabular}

Esquema 1. Célula potenciométrica: $\mathrm{Ag}|\mathrm{AgCl}| \mathrm{Cl}^{-}$ vs. Pt $|\mathrm{Hg}| \mathrm{Hg}_{2} \mathrm{Ox} \mid$ Grafite

A célula potenciométrica (Esquema 1) foi preparada acrescentando-se: a) $15,000 \mathrm{~mL}$ de $\underline{Y}=$ $\mathrm{NaClO}_{4}$ ou $\mathrm{NaNO}_{3} 0,700 \mathrm{~mol} \mathrm{~L}^{-1}$; b) $15,000 \mathrm{~mL}$ de $\underline{\mathrm{Y}}=\mathrm{NaClO}_{4}$ ou $\mathrm{NaNO}_{3} 0,700 \mathrm{~mol} \mathrm{~L}^{-1}$ e ponta de espátula (cerca de $10 \mathrm{mg}$ ) de $\mathrm{Hg}_{2} \mathrm{Ox}$; c) $15,000 \mathrm{~mL}$ de $\mathrm{Na}_{2} \mathrm{Ox} 5,910^{-2} \mathrm{~mol} \mathrm{~L}^{-1}$ em força iônica (I) 0,700 mol L-1, ajustada com $\underline{Y}=\mathrm{NaClO}_{4}$ ou $\mathrm{NaNO}_{3}$, perfazendo-se um total de seis experimentos.

$\mathrm{O}$ eletrodo de referência utilizado, em todas as células, foi o $\mathrm{Ag}|\mathrm{AgCl}| \mathrm{Cl}^{-}$de procedência "Metrohm", no 6.0726.100 de dupla junção (associado a uma ponte salina vertical).

\section{Medidas potenciométricas}

Foi utilizado o método potenciométrico visando o estudo do efeito da pressão na prensagem da pastilha sensora. As medidas potenciométricas para a determinação das curvas analíticas em meio de $\mathrm{NaClO}_{4}$ ou $\mathrm{NaNO}_{3}$ foram realizadas conforme 
descrito em trabalho anterior [4], utilizando o titulador automático "Titroprocessor Metrohm", mod. 670 (com precisão de $\pm 0,1 \mathrm{mV}$ ), acoplado a buretas de pistão automáticas "Metrohm", mod. 665 (com precisão de $\pm 0,001 \mathrm{~mL}$ ) e a célula potenciométrica do Esquema 1, termostatizada $(25,0 \pm 0,1)^{\circ} \mathrm{C}$.

As células potenciométricas foram preparadas da seguinte maneira: acrescentou-se $15,000 \mathrm{~mL}$ de $\mathrm{NaClO}_{4}$ ou $\mathrm{NaNO}_{3}$, em I $=0,700 \mathrm{molL}^{-}$ ${ }^{1}$, uma ponta de espátula (cerca de $10 \mathrm{mg}$ ) de $\mathrm{Hg}_{2} \mathrm{Ox}$ e $0,500 \mathrm{~mL}$ de solução de $\mathrm{Na}_{2} \mathrm{Ox} 0,100 \mathrm{molL}^{-1}$, na força iônica de medida - "solução titulante". Esta solução foi deixada sob agitação até saturação da solução de medida com $\mathrm{Hg}_{2} \mathrm{Ox}$ (cerca de 30 minutos); em seguida foram adicionados automaticamente pelo titulador potenciométrico incrementos constantes de $0,250 \mathrm{~mL}$ a cada $30 \mathrm{se}$ medida a força eletromotriz da célula, até um total de $30,000 \mathrm{~mL}$ da "solução titulante".

\section{Microscopia Ótica}

A microscopia ótica foi utilizada para verificar a morfologia das superfícies das pastilhas sensoras dos eletrodos utilizados nas experiências. Utilizou-se o microscópio ótico Leica DM R, com aquisição das micrografias através do software Leica Qwin - Image Processing and Analyser System (Leica Imaging Systems Ltd, Cambridge, England, 1996).

\section{Espectroscopia no Infravermelho}

A espectroscopia no infravermelho foi usada para verificar a composição das superfícies das pastilhas sensoras dos eletrodos utilizados nas experiências. $\mathrm{O}$ aparelho usado foi o IMPACT 400 - IR/FT Spectrometer e os espectros foram obtidos em pastilha de $\mathrm{KBr}$, contendo as amostras em pó.

\section{Resultados e Discussão}

\section{Influência do meio iônico no potencial da célula}

As Figuras 2 e 3 mostram as seis curvas de potencial em função do tempo em três situações para cada eletrólito: $\mathrm{NaClO}_{4}$ (Figura 2) e $\mathrm{NaNO}_{3}$ (Figura 3). A concentração de $\mathrm{Na}_{2} \mathrm{Ox}=5,910^{-2} \mathrm{~mol}$ $\mathrm{L}^{-1}$ refere-se à concentração próxima da intermediária das curvas analíticas em meio de $\mathrm{NaClO}_{4}$ e em meio de $\mathrm{NaNO}_{3}{ }^{3}$, para $\mathrm{I}=0,700 \mathrm{~mol} \mathrm{~L}$.

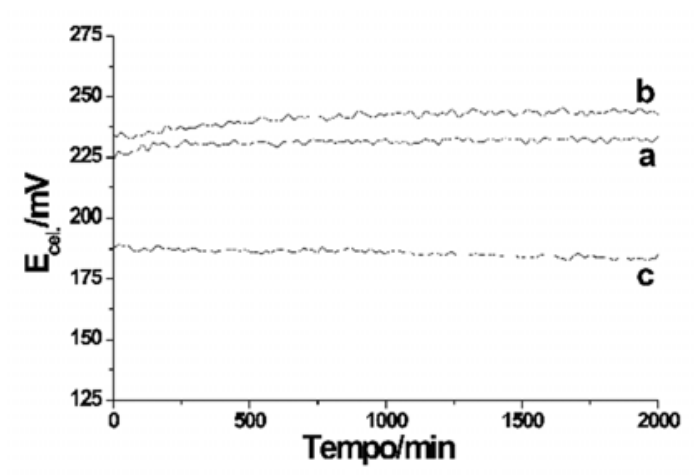

Figura 2. Influência do meio iônico no potencial da célula utilizando os eletrodos $\mathrm{Ag}|\mathrm{AgCl}| \mathrm{Cl}^{-}$vs. $\mathrm{Pt}|\mathrm{Hg}| \mathrm{Hg}_{2} \mathrm{Ox} \mid$ Grafite, em função do tempo e em meio de: a) solução de $\mathrm{NaClO}_{4}, \mathrm{I}=0,700 \mathrm{~mol} \mathrm{~L}-1$, b) solução de $\mathrm{NaClO}_{4}$, $\mathrm{I}=0,700 \mathrm{~mol} \mathrm{~L}^{-1}+$ ponta de espátula (cerca de $10 \mathrm{mg}$ ) de $\mathrm{Hg}_{2} \mathrm{Ox}$ e c) solução de $\mathrm{Na}_{2} \mathrm{Ox} 5,910^{-2} \mathrm{~mol} \mathrm{~L}^{-1}, \mathrm{I}=0,700 \mathrm{~mol}$ $\mathrm{L}^{-1}\left(\mathrm{NaClO}_{4}\right)$.

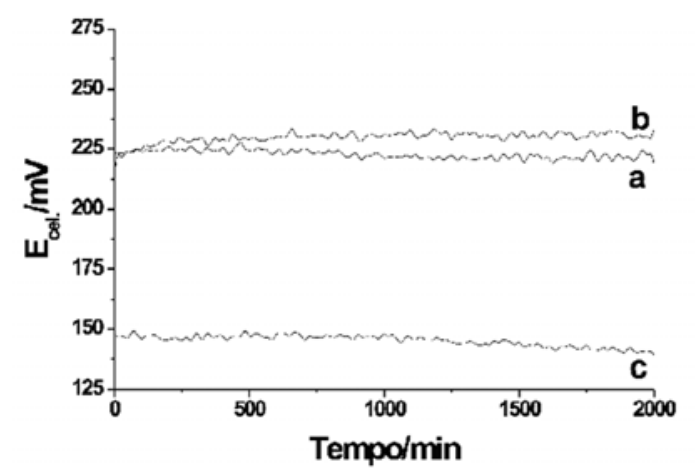

Figura 3. Influência do meio iônico no potencial da célula utilizando os eletrodos $\mathrm{Ag}|\mathrm{AgCl}| \mathrm{Cl}^{-} v s$. $\mathrm{Pt}|\mathrm{Hg}| \mathrm{Hg}_{2} \mathrm{Ox} \mid$ Grafite, em função do tempo e em meio de: a) solução de $\mathrm{NaNO}_{3}, \mathrm{I}=0,700 \mathrm{~mol} \mathrm{~L}{ }^{-1}$, b) solução de $\mathrm{NaNO}_{3}$, $\mathrm{I}=0,700 \mathrm{~mol} \mathrm{~L}^{-1}+$ ponta de espátula (cerca de $10 \mathrm{mg}$ ) de $\mathrm{Hg}_{2} \mathrm{Ox} \mathrm{e} \mathrm{c)} \mathrm{solução} \mathrm{de} \mathrm{Na}_{2} \mathrm{Ox} 5,910^{-2} \mathrm{~mol} \mathrm{~L}^{-1}, \mathrm{I}=0,700 \mathrm{~mol}$ $\mathrm{L}^{-1}\left(\mathrm{NaNO}_{3}\right)$.

Analisando as curvas da Figura 2 e 3 observa-se que o comportamento das curvas $\underline{a}$ e $\underline{b}$ é semelhante em meios de eletrólitos de suporte puros e também com adição de cerca de $10 \mathrm{mg}$ de $\mathrm{Hg}_{2} \mathrm{Ox}$. Entretanto, em meio de solução de $\mathrm{Na}_{2} \mathrm{Ox}, \mathrm{o}$ comportamento das curvas $\underline{\mathrm{c}}$, das respectivas figuras é diferente, apresentando um decréscimo um pouco mais acentuado na curva da Figura 3, em 
função do tempo.

As micrografias das pastilhas sensoras empregadas nos experimentos referentes às curvas $\underline{a}$ e $\underline{b}$ das Figuras 2 e 3 não mostraram alterações significativas nas suas superfícies em relação à micrografia da pastilha sensora sem uso (Figura 4.I). Enquanto que as micrografias das pastilhas sensoras empregadas nos experimentos referentes às curvas $\underline{c}$ das Figuras 2 e 3 estão apresentadas respectivamente, nas Figuras 4.II e 4.III .
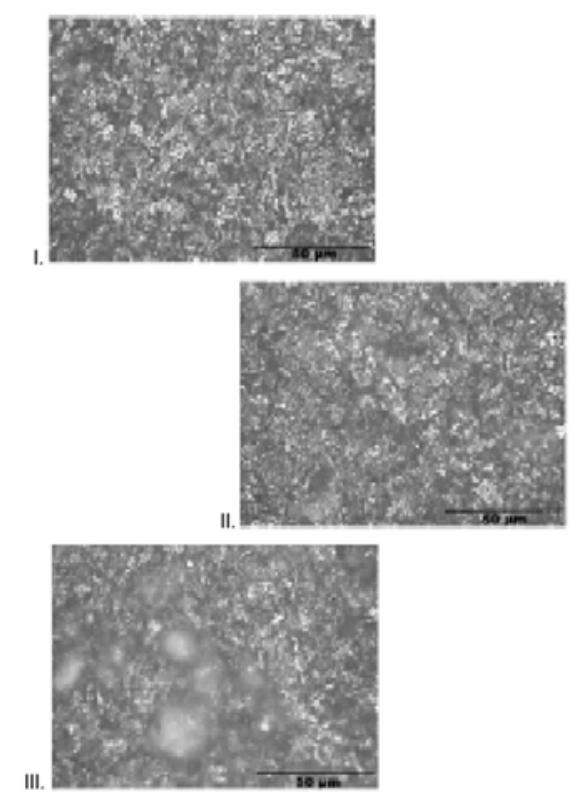

Figura 4. Micrografias das pastilhas sensoras: I) Após prensagem (sem uso) e utilizadas nas experiências sobre a influência do meio iônico, em solução de $\mathrm{Na}_{2} \mathrm{Ox} 5,9$ $10^{-2} \mathrm{~mol} \mathrm{~L}^{-1}$ em força iônica $0,700 \mathrm{~mol} \mathrm{~L}^{-1}$ ajustada com: II) $\mathrm{NaClO}_{4}$ e III) $\mathrm{NaNO}_{3}$.

Analisando a micrografia da Figura 4.II observa-se que esta é semelhante a da Figura 4.I, evidenciando que o meio de perclorato contendo $\mathrm{Na}_{2} \mathrm{Ox}$ não influencia na morfologia superficial da pastilha sensora, pois a morfologia permanece semelhante à da pastilha antes de ser usada. Entretanto, a micrografia da Figura 4.III mostra uma alteração morfológica na superfície da pastilha sensora quando usada em meio de $\mathrm{NaNO}_{3}$ contendo $\mathrm{Na}_{2} \mathrm{Ox}$. A alteração observada sugere a ocorrência de formação de gotas de mercúrio metálico na superfície da referida pastilha. Este comportamento poderia ser explicado pela existência de uma provável interação entre o íon nitrato e o íon oxalato, com eventual formação de íon nitrito, onde este reduziria o íon mercuroso a mercúrio metálico, com regeneração do íon nitrato.

\section{Espectros na região do Infravermelho da pastilha sensora, da pasta $\mathrm{Hg}\left|\mathrm{Hg}_{2} \mathrm{Ox}\right|$ Grafite e do grafite em pó}

$\mathrm{Na}$ Figura 5 estão apresentados os espectros na região do Infravermelho da superfície da pastilha sensora utilizada em meio de $\mathrm{NaNO}_{3}$ contendo $\mathrm{Na}_{2} \mathrm{Ox}$, da pasta $\mathrm{Hg}\left|\mathrm{Hg}_{2} \mathrm{Ox}\right|$ Grafite sem prensar e do grafite em pó (Merck, extra pure), visando obter informações sobre as amostras analisadas

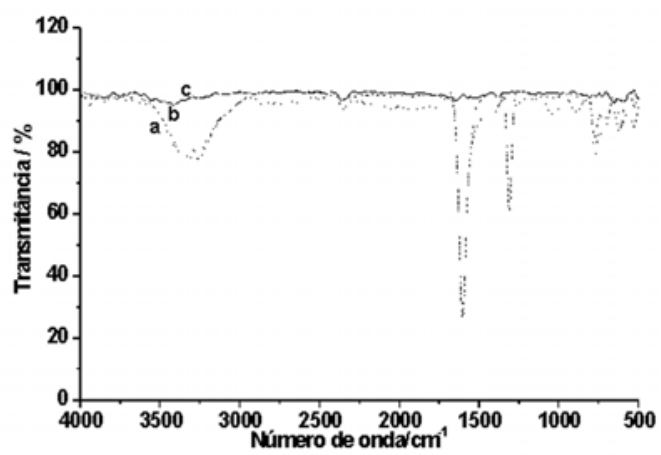

Figura 5. Espectros na região do Infravermelho em pastilhas de $\mathrm{KBr}$ contendo amostras em pó de: a) superfície da pastilha sensora utilizada nos experimentos, b) pasta $\mathrm{Hg}\left|\mathrm{Hg}_{2} \mathrm{Ox}\right|$ Grafite sem prensar e c) grafite (Merck, extra pure).

Analisando os espectros da Figura 5 observa-se que os espectros a e $\underline{\text { b }}$ são praticamente coincidentes e que as pequenas diferenças observadas devem-se, provavelmente, à variação na concentração da amostra nas pastilhas de $\mathrm{KBr}$. As bandas observadas nestes espectros podem ser atribuídas aos estiramentos característicos de carboxilatos, isto é do oxalato presente. O espectro c da referida figura não apresenta bandas características de outros compostos orgânicos que poderiam estar adsorvidos na superfície do grafite, evidenciando sua pureza.

\section{Efeito da pressão de prensagem da pastilha sobre sinal potenciométrico}

A pressão de prensagem de pastilhas 
sensoras de eletrodos potenciométricos pode influir na resposta do eletrodo [14].

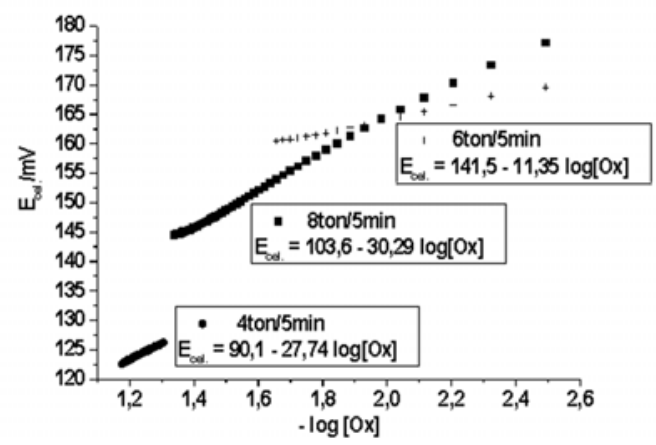

Figura 6. Efeito da pressão de prensagem da pastilha sensora sobre o potencial do eletrodo indicador, em força iônica $0,700 \mathrm{~mol} \mathrm{~L}^{-1}$ ajustada com $\mathrm{NaClO}_{4}$.

As Figuras 6 e 7 mostram as curvas analíticas obtidas com as células descritas anteriormente, onde os eletrodos foram construídos conforme descrito em trabalho anterior [4], variando-se a pressão de prensagem da pastilha sensora, conforme indicado nas referidas figuras.

As curvas das Figuras 6 e 7 mostram que a variação na pressão para obtenção das pastilhas sensoras exerce efeito significativo tanto no potencial da célula, como no coeficiente angular das curvas e também no intervalo linear considerado para cada ajuste. Quando a pastilha sensora é prensada com pressões de 4,6 e 8 toneladas-força/ $\mathrm{cm}^{2}$ o eletrodo em meio de $\mathrm{NaClO}_{4}$ apresenta comportamento nernstiano para valores de pressão de 4 e 8 toneladas-força $/ \mathrm{cm}^{2}$, além de um maior

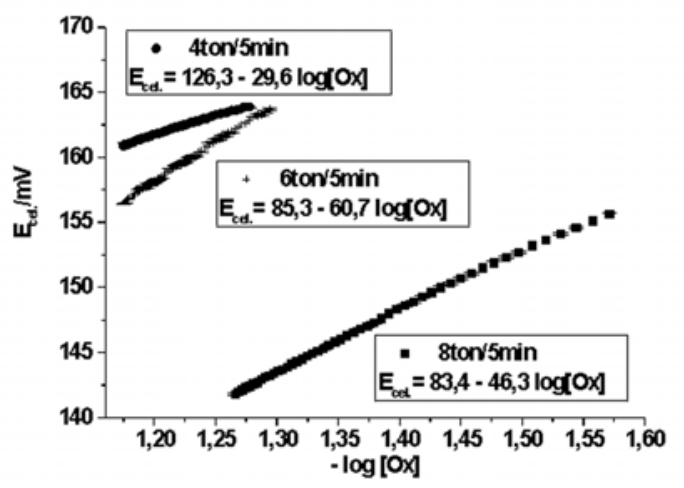

Figura 7. Efeito da pressão de prensagem da pastilha sensora sobre o potencial do eletrodo indicador, em força iônica $0,700 \mathrm{~mol} \mathrm{~L}^{-1}$ ajustada com $\mathrm{NaNO}_{3}$. intervalo linear quando se aplica uma pressão de 8 toneladas-força $/ \mathrm{cm}^{2}$ na prensagem da pastilha sensora. Entretanto, se o eletrodo é utilizado em meio de $\mathrm{NaNO}_{3}$ o comportamento nernstiano só é observado quando a pasilha sensora é prensada a 4 toneladas-força $/ \mathrm{cm}^{2}$.

\section{Conclusões}

O eletrodo Pt $|\mathrm{Hg}| \mathrm{Hg}_{2} \mathrm{Ox} \mid$ Grafite em meio de $\mathrm{NaNO}_{3}$, apresenta uma diminuição na sensibilidade em função do tempo de uso, resultante da alteração da superfície da pastilha sensora, onde, provavelmente, o mercúrio(I) do oxalato mercuroso se reduz a mercúrio metálico (induzido pelo ín oxalato em meio de $\mathrm{NaNO}_{3}$ ).

Portanto, a utilização do referido eletrodo fica prejudicada em meio de $\mathrm{NaNO}_{3}$, durante um período de tempo prolongado. Para que este eletrodo possa ser usado neste meio, os procedimentos experimentais deverão ser conduzidos com cautela, isto é, o eletrodo deverá ficar imerso em solução de $\mathrm{NaNO}_{3}$ contendo $\mathrm{Na}_{2} \mathrm{Ox}$, somente durante o tempo de execução do experimento.

\section{Agradecimentos}

Os autores agradecem à FAPESP e a FUNDUNESP os auxílios à pesquisa e à CAPES a bolsa concedida.

VILLAFRANCA, A. C.; MORAES, M. de; PEZZA, L. Evaluation and characterization of the sensor pellet of an electrode of the second kind sensitive to oxalate.

\begin{abstract}
The $\mathrm{Pt}|\mathrm{Hg}| \mathrm{Hg}_{2} \mathrm{Ox} \mid$ Graphite $(\mathrm{Ox}=$ oxalate ion) electrode was studied using $\mathrm{NaClO}_{4}$ as the support electrolyte. Preliminary experiments were carried out using $\mathrm{NaNO}_{3}$ as support electrolyte, following the same methodology as the previous work, and it was verified that there was a decrease in the electrode sensitivity over its usage time. In order to understand the behavioral difference of the electrode in $\mathrm{NaClO}_{4}$ and $\mathrm{NaNO}_{3}$ media, experiments were carried out to obtain potential vs time curves to verify the behavior as a function of
\end{abstract}


time and also in different ionic media. The surface morphology of the sensor pellet used in $\mathrm{NaNO}_{3}$ media presented alteration, which suggests that this media favors reactions on the pellet surface when it comes into contact with the solution.

Key words: electrode of the second kind, oxalate, perchlorate, nitrate

\section{Referências bibliográficas}

[1] BRAUER, G. Handbook of preparative inorganic chemistry. New York: Academic Press, 1963. p. 27-29, 1120.

[2] CASELLA, I. G.; ZAMBONIN, C. G.; PRETE. F. Liquid chromatography with eletrocatalytic detection of oxalic acid by a palladium-based glassy carbon. J. Chrom. A, v. 833 , p. $75-82,1999$.

[3] CAVAlHeiro, A. C. V. Construção, estudo analítico e aplicação físico-química de um eletrodo de segunda espécie, sensivel ao ion oxalato, em meio aquoso. Araraquara, 2001. 118f. Dissertação (Mestrado em Química) - Instituto de Química, Universidade Estadual Paulista, Araraquara, 2001.

[4] CAVAlheiro, A. C. V.; MORAeS, M.; PEZZA, L. Construção e aplicação físico-química de um eletrodo de segunda espécie, sensível ao íon oxalato, em meio aquoso. Ecl. Quim., v. 25, p. 123-135, 2000.

[5 ] FERRI, D.; MANFREDI, C.; SALVATORE, F.; TRIFUOGGI, M.; VASCA, E.; FINA, E. On a reversible electrode for potentiometric determination of oxalate. Ann. Chim., v. 88, p. 111-120, 1998.

[6] HODGKINSON, A. Oxalic acid in biology and medicine. London: Academic Press, 1977. p. 22-61.

[7] JAMES, H.; CARMACK, G.; FREISER, H. Coated wire ion selective electrodes. Anal. Chem., v. 44, n. 4, p. 856$857,1972$.

[8] MUÑOZ, J. A.; CAMPAÑA, A. M. G.; BARRERO, F. A. Effect of cationic micelles on the formation of the complex oxalate-Alizarin Red S-Zr(IV). Application to the sensitive fluorescence determination of oxalate ion. Talanta, v. 47, p. 387-399, 1998.

[9] OLIVEIRA NETO, G.; TUBINO, M.; GODINHO, O. E. S.; KUBOTA, L. T.; FERNANDES, J. R. Oxalate determination in urine using na immobilized enzyme on Sorghum vulgare seeds in flow injection conductimetric system. J. Braz. Chem. Soc., v. 8, n. 1, p. 47-51, 1997.

90
[10] STEFAN, R. I.; DRAGHICI, I.; BAIULESCU, G. E. Determination of urinary oxalate-selective membrane electrodes. Sensors Actuators B-Chem., v. 65, n. 1-3, p. 250252, 2000 .

[11] VASCA, E.; CARUSO, T.; IULIANO, M.; MANFREDI, C.; FONTANELLA, C.; TRIFUOGGI, M. Studies on metal oxalate complexes. I. Validation of the reversibilyti of the $\mathrm{Hg}, \mathrm{Hg}_{2} \mathrm{C}_{2} \mathrm{O}_{4(\mathrm{~s})}$ electrochemical couple. Ann. Chim., v. 90, n. 3-4, p. 181-192, 2000.

[12] VLASOV, Y. G.; KOLODNIKOV, V. V.; ERMOLENKO, Y. E.; MIKHAILOVA, S. S. Chemical sensors and the development of potentiometric methods for analysis of liquid media. J. Anal. Chem., v. 51, n. 8, p. 741-752, 1996.

[13] WU, F.; HE, Z.; LUO, Q.; ZENG, Y. HPLC determination of oxalaic acid using tri (1,10-phenantroline) ruthenium(II) chemiluminescence - application to the analysis of spinach. Food Chem., v. 65, p. 543-546, 1999.

[14] ZAREH, M. M.; AMIN, A. S. Characterization of a novel tellurite selective electrode. Microchem. J., v. 56, p. 276-284, 1997.

Recebido em 28.02.2003.

Aceito em 23.04.2003 